\title{
Protection of Diol as Acetonide Using Acetone and Cation Exchange Resin as a Catalyst.
}

\author{
B. B. Bahule*a, Y. M. Nandurkar ${ }^{\mathrm{a}}$ \\ ${ }^{a}$ Department of Chemistry, Nowrosjee Wadia College, Pune, India.
}

\begin{abstract}
The selective introduction of acetonide as a protecting group for various diols has been achieved in an excellent yield under mild reaction conditions. The diol is transformed into acetonide with acetone and a cation exchange resin in solvent free condition.
\end{abstract}

Keywords: Acetonide, diols, resins.

\section{Introduction}

Protection could be regarded as a special instance of a combine chemo and region selectivity since it embrace aspects of both. It is implicated when a reaction selectivity at one functional group is needed in the presence of other functional group. When the principles of chemo selectivity are not applicable, the accompanying functional group must be protected [1]. Hydroxyl group of primary secondary, tertiary, allylic or alicyclic alcohol may be protected by conversion into an ester [2], a silyl ether [3], an ester [4] or acetals and ketals [5]. The most important method for the protection 1.2 diols or 1,3 diols is to convert them into a cyclic acetals or ketals. When the alcohols do not carry base labile group, they can be converted into their methyl ethers with suitable methylating agents i.e. diazomethane, methyl iodide etc. Methyl ethers are stable to acidic and basic conditions but deprotection of these to regenerate alcohol is a difficult task. The alcohols can be protected as silyl ethers with trimethylsilyl chloride. This protecting group is quite stable to most of the reaction conditions except tetrabutyl ammonium fluoride [6]. Alcohols can be esterified and the esters can be hydrolysed to regenerate alcohols.

\section{Present Work}

In this letter, we wish to report a mild efficient and ecofriendly method to protect 1,2 or 1,3 diols as their acetonides with acetone and a cation exchange resin. The reaction can be carried out in toluene or without toluene as a solvent. The selectivity and versatility of the of the thermal solvent free reaction was further confirmed by applying it to various examples as shown in table 1 [7]

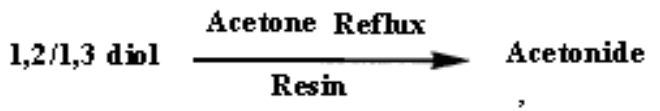

Table 1 Resin catalyzed acetonide formation of $1,2 / 1,3 \mathrm{diols}$

\begin{tabular}{|c|c|c|c|c|c|}
\hline S. No. & $1,2 / 1,3$ diol & \multicolumn{2}{|c|}{ Stirring in Toluene } & \multicolumn{2}{c|}{ Reflux in Acetone } \\
\hline & & Time (hr) & Yield $(\%)$ & Time (hr) & Yield (\%) \\
\hline 1 & Resorcinol & 10 & 76 & 1.5 & 99 \\
\hline 2 & Catechol & 5 & 75 & 1.3 & 87 \\
\hline 3 & Propane 1,3 diol & 10 & 77 & 1.5 & 95 \\
\hline 4 & Glycerol & 8 & 94 & 1.5 & 95 \\
\hline 5 & Cholestane, 2,3 diol & 10 & 95 & 2.5 & 96 \\
\hline 6 & Cxclopexane, 1,2 diol & 8 & 90 & 1.5 & \\
\hline
\end{tabular}

\section{Experimental Section}

The acetonides are prepared by two methods. The first method involves the use of Toluene as a solvent and 1,2/1,3 diol, acetone, resin as other chemical. The reaction mixture is stirred for 5-10 hrs. at room temperature and the solvent was removed by simple or vaccum distillation after the removal resin by filteration. The crude products were purified by neutral alumina column chromatography. The alternate method does not require toluene as a solvent. The acetonide is prepared in small reaction time when 1,2/1,3 diol, acetone and resin are subjected to reflux. The reaction mixture is filtered to recover the resin without the loss of its chemical reactivity. This method has simplicity in operation and mild reaction conditions tolerable to acid functionalities. It is cost efficient method, ecofriendly and better than the homogeneous counterpart. The products were characterized by IR and PMR spectroscopy. 
Spectral data for acetonide of catechol (Sr.No.2 of Table 1)

IR : $1263.3 \mathrm{~cm}^{-1} \mathrm{C}-\mathrm{O}$ stretching frequency.

$748.3 \mathrm{~cm}^{-1} \mathrm{C}-\mathrm{H}$ out of plane bending vibrations for $\mathrm{Ar}-\mathrm{CH}$.

PMR: $\mathrm{CDCl}_{3}(\mathrm{ppm}) 2.15 \mathrm{~s}, 6 \mathrm{H}, 7.2 \mathrm{dd}, 2 \mathrm{H}, 7.6 \mathrm{dd}, 2 \mathrm{H}$.

\section{Acknowledgements}

We wish to acknowledge, Principal, Nowrosjee Wadia College, Pune for providing necessary infrastructure and support for research work. We are also thankful to HOD, Department of Chemistry for constant encouragement.

\section{References}

[1] Green, I. W.; Wuts, P.G.M. Protective groups in Organic Synthesis, $3^{\text {rd }}$ edition, Wiley and Sons, New York 1999,116

[2] Kartha, K.P.R.; Tet. Lett. 1986, 27, 3415

[3] Cooper, B.E.; Chem. Ind. 1978,794

[4] House, H.O.; Czuba, L.J.M.; Olmstead Gall, Olmstead, H.D., J. Org. Chem. 1969,34,2324

[5] Kumar, P.; Dinesh, C.U.; Reddy, R. S.; Pande, B. Synthesis 1993,1069

[6] West, R.; J. Am. Chem. Soc. 1958,80,3246

[7] The cation exchange resins were obtained from Ion exchange India Ltd. Amberlite, Tulsion, Indion, Amberlyst were used as a catalyst for acetonide preparation. 